\title{
Analysing the growth of OpenStreetMap networks
}

\author{
Padraig Corcoran ${ }^{\mathrm{a}, *}$, Peter Mooney ${ }^{\mathrm{b}}$, Michela Bertolotto ${ }^{\mathrm{a}}$ \\ a School of Computer Science and Informatics, University College, Dublin, Ireland \\ ${ }^{\mathrm{b}}$ Department of Computer Science, National University of Ireland, Maynooth, Ireland
}

\section{A R T I C L E I N F O}

\section{Article history:}

Received 3 September 2012

Accepted 15 January 2013

Available online 4 February 2013

\section{Keywords:}

OpenStreetMap

Complex network

Spatial process

\begin{abstract}
A B S T R A C T
In this article we analyse the growth of OpenStreetMap (OSM) representations for three street networks in Ireland. In each case we demonstrate the growth to be governed by two elementary spatial processes of densification and exploration which are responsible for increasing the local density of the network and expanding the network into new areas respectively. We also examine summary statistics describing each network topology and show these to be a consequence of the same processes. This represents the discovery of a novel link between different aspects of the growth.
\end{abstract}

(c) 2013 Elsevier B.V. All rights reserved.

\section{Introduction}

The term Volunteered Geographic Information (VGI) describes the practice of large numbers of citizens, mostly with no formal training, engaging in the creation of spatial data (Goodchild, 2007). VGI has proven to be an effective means of acquiring timely and detailed geographical information (Goodchild and Li, 2012). Consequently it is an important source of spatial information in a variety of applications from transportation routing to emergency management (Goodchild and Glennon, 2010). The OpenStreetMap (OSM) project was founded in the year 2004 and is one of the best known sources of VGI (Mooney et al., 2010). Generally OSM contributors operate without central coordination or strict data frameworks, although coordination may be witnessed at times in the form of mapping parties. As such the OSM mapping process is considered by many to be complex and unorthodox in the sense that it contrasts greatly with that of national mapping agencies (Haklay et al., 2010). The contribution of new data to OSM can be done in a number of ways (Neis and Zipf, 2012). The most common approach

\footnotetext{
* Corresponding author. Tel.: +35317162894.

E-mail address: padraig.corcoran@ucd.ie (P. Corcoran).
} 
is to record data using a GPS receiver and edit this using one of the many freely available editors. If aerial imagery is available, data may also be manually digitised from this. In recent years there have been a number of cases where freely available data has been bulk imported into OSM. For example the complete road network for the USA was imported into OSM from the TIGER dataset in 2007 and 2008.

The process by which OSM contributions map the physical environment is inherently spatial and therefore may be subject to spatial analysis and modelling. For example, most contributions map only their local community and this area may be located and its extent measured (Neis and Zipf, 2012). A number of authors have noted the need for such analysis and modelling as it offers many potential benefits (Kuhn, 2007; Mulligann et al., 2011). Firstly it could be used as a tool for central coordination and the targeting of contributors with specific mapping tasks (Panciera et al., 2010). OSM does not carry any of the data quality guarantees associated with data from national mapping agencies. If an accurate model of the OSM mapping process was available it could be used to classify the quality of the data in question (Mulligann et al., 2011). The research objective of this article is to statistically analyse and model the growth of OSM street network representations and in turn provide new insights into the dynamics of OSM mapping. Specifically this work makes two contributions. Firstly we demonstrate the growth of the OpenStreetMap (OSM) representation for three street networks in Ireland to be governed by the two elementary spatial processes of exploration and densification. Secondly, we show how these processes explain summary statistics describing each network topology. This represents the discovery of a novel link between different aspects of the network growth.

The layout of this paper is as follows. Section 2 reviews related works on the topic of spatial analysis and modelling. Section 3 describes the geographical regions for which we examine the growth of the corresponding OSM street networks. This section also describes the methodology employed for converting the OSM data to a network representation. Section 4 presents results. In the final part of this paper, Section 5, we draw conclusions from this work and discuss possible future research directions.

\section{Related works}

In this section we review related works in the domain of spatial analysis and modelling. Specifically, in each of the following two sub-sections we concentrate on those works related to the topics of spatial analysis of OSM and street networks.

\subsection{OpenStreetMap}

OSM data and it contributors have been the subject of significant spatial analysis and modelling. Many studies have analysed the quality of OSM data. Haklay (2010) and Girres and Touya (2010) performed a spatial analysis of the quality of OSM street network representations in the UK and France respectively through a comparison to ground-truth data obtained from the corresponding national mapping agency. Both studies found that on average the quality of the data was reasonably good but exhibited significant spatial heterogeneity. Neis et al. (2012) analysed how the quality of the OSM street network in different regions of Germany changed between the years 2007-2011. The authors found that, as of 2011, on average the OSM street network representation exceeds the information contained in a proprietary dataset by $27 \%$.

Given that OSM data quality exhibits spatial heterogeneity many works have attempted to model different aspects of data quality towards quality assessment and improvement. Haklay et al. (2010) attempted to model the relationship between OSM data quality for a region and the number of active contributors in that region. He found that beyond 15 contributors per square kilometre the accuracy of the OSM street networks was high. In works related to this Roick et al. (2013) and Trame and Keßler (2011) analysed the spatial distribution of a number of OSM variables including total number of edits and number of contributors. Neis and Zipf (2012) performed an in-depth analysis of OSM contributor activity. This included an analysis of contributor location of residence and the extend of OSM areas they edited. Mashhadi et al. (2013) attempted to model the spatial relationship between OSM coverage in London and a number of variables. The authors discovered a strong correlation 
between OSM coverage and population density. A less, but significant, correlation was discovered between OSM coverage and a number of socio-economic variables. Mulligann et al. (2011) combined semantic similarity with concept variograms and spatial point pattern analysis to model interaction characteristics of features in OSM towards data quality assessment and improvement.

\subsection{Street networks}

Since the discovery that a variety of complex networks exhibit small-world properties, characterised by clustering and shortcuts, there has been a significant interest in the statistical analysis of complex networks (Watts and Strogatz, 1998). Complex networks are generally considered as lying in an abstract space, where the location of vertices has no particular meaning. For many types of networks, such as citation networks, this consideration is justifiable. However, there are many network types where the location of vertices is particularly important as direct connections between vertices can depend on constraints such as the cost associated with the length of edges. These types of networks are known as spatial networks and examples include power grids and street networks (Barthelemy, 2011). The constraints to which spatial networks are subject have dramatic effects on their corresponding topological structure and statistical properties (Costa et al., 2007). For example, the degree of vertices in spatial networks are practically limited and the average path length is generally much larger than for other types of networks with a comparable number of vertices (Chan et al., 2011). Consequently spatial networks exhibit statistical properties which are distinct from other types of networks.

Many studies have analysed and modelled the statistical properties of transportation networks which are a specific type of spatial network. It has been demonstrated that some street networks exhibit scale-free and small-world properties (Porta et al., 2006; Ferber et al., 2009) while other street networks exhibit small-world properties but not scale-free properties (Jiang and Claramunt, 2004). Buhl et al. (2006) analysed the topological patterns of a large number of street networks in different countries. Derrible and Kennedy (2010) examined the structure of 33 metro systems in terms of state, form and structure. Masucci et al. (2009) performed a statistical analysis of the London street network relative to three models. Crucitti et al. (2006) compared the statistical properties of street networks corresponding to different classes of cities such as planned and self-organised.

In an effort to better understand the statistical properties exhibited by transportation networks many studies have analysed and modelled the corresponding growth of such networks. Erath et al. (2009) presented an analysis of the Swiss street and rail network growth during the period 1950-2000. Barthelemy and Flammini (0000) proposed a model of network growth based on local optimisation combined with ideas previously proposed in studies of leaf pattern formation. The authors demonstrated their model generates networks with similar statistical properties to the street network of Dresden city. Ferber et al. (2009) proposed a model of network growth where new candidate edges are generated using random walks and these are subsequently added to the network if they do not self-intersect. The authors demonstrated their model generates networks with similar statistical properties to the public transportation networks of a number of cities including Sydney and Paris. Xie and Levinson (2009) proposed a model which adds a set of new edges but subsequently either removes or confirms individual elements of this set. This model considers the context of the growth which allows for the interaction between demand (travelers) and supply (transportation network). The authors validated the model using historical data from the Indiana interurban network. Strano et al. (2012) demonstrated the growth of a street network located in a region north of Milan Italy to be governed by two elementary spatial processes referred to as densification and exploration. The model of Strano et al. (2012) will be described in detail in Section 4.2.

Methods developed for the study of spatial networks may also be applied to study the representation of such networks in OSM. Given that both types of network are spatial, the networks in question and their growth many potentially exhibit similar statistical properties. However, due to the fact that the processes underlying the growth of both types of networks are very different this may not be the case. For example, the growth of street networks in a country is a function of decisions made by its government. This is generally not the case for their corresponding representations in OSM which are a function of possibly uncoordinated sporadic contributors. To date only a couple of works 
have examined the properties of street networks in OSM using techniques from the domain of network theory. Neis et al. (2012) studied the growth of OSM street networks in terms of street length, number of topological errors and street semantics. In a related work Corcoran and Mooney (2013) studied the growth of OSM street networks in terms metric and topological characteristics.

\section{Study regions \& network representation}

In this article we analyse the growth of three OSM street networks corresponding to the urban regions of Maynooth, Waterford and Wicklow located in Ireland. Maynooth is a University town in Ireland located $24 \mathrm{~km}$ west of Dublin and has a population of over 1100 . The area of this region is $10.5 \mathrm{~km}^{2}$. Waterford is city located in the South-East of Ireland at the head of Waterford Harbour and has a population of over 50,000 . The area of this region is $99.3 \mathrm{~km}^{2}$. Finally Wexford is town located near the South-East corner of Ireland and has a population of over 9000. The area of this region is $33.8 \mathrm{~km}^{2}$. None of the three chosen regions were subject to bulk data imports. For each region the street network corresponding to the first day of each month contained within the period 01-11-2007-01-10-2011 inclusive was extracted using the methodology described in Mooney and Corcoran (2011). This provided a total of 48 street network versions for each region. This period was chosen as it corresponded to a growth of each network from containing little or no street network detail to a highly detailed street network.

The OSM database contains points, lines and polygons which are commonly referred to as nodes, ways and areas respectively. OSM points consist of a pair of latitude and longitude coordinates, additional information in the form of tags and a unique identifier (ID). OSM lines consist of a sequence of OSM points, additional information in the form of tags and a unique ID. OSM polygons are identical to OSM lines except that they enclose an area. A street network in OSM is represented by a set of lines.

Graph theory provides a natural environment to study street networks. A graph $G=(V, E)$ consists of a set of vertices $V$ and a set of edges $E$ which are unordered pairs of vertices. If we assume that the vertices are the street intersections and the extremes of dead end streets, and the edges the street fragments connecting these vertices, we obtain a primary representation of the street network. On the other hand, if we assume the vertices are streets and two vertices are connected whenever the streets they represent intersect, we obtain a dual representation of the street network (Masucci et al., 2009). It was noted by Crucitti et al. (2006) that a dual representation corresponds to an abandonment of metric distance; a street is a single node irrespective of its length. In order to study the growth of the OSM street networks we converted each network version $i$ to its corresponding primary representation denoted $G_{i}=\left(V_{i}, E_{i}\right)$. It was determined that two streets intersect if their corresponding OSM line representations share a common OSM point. Recently a number of authors have proposed to study the growth of networks using only a single graph representation which encodes the temporal properties of growth (Holme and Saramaki, 2012). However such approaches are still very new and underdeveloped.

The growth of the OSM street network corresponding to the Maynooth region is illustrated in Fig. 1(a)-(f) which shows the state of the network on the dates 01-11-07, 01-02-09, 01-03-09, 0107-09, 01-09-09 and 01-10-11 respectively. The number of vertices, denoted $\left|V_{i}\right|$, contained in these networks is $25,785,1475,2061,2764$ and 3438 respectively.

\section{Results}

This section is divided in three sub-sections where each examines a different aspect of OSM street network growth. The first sub-section examines the rate of network growth. It was recently demonstrated by Strano et al. (2012) that the growth of a street network may be governed by two elementary spatial processes known as densification and exploration. In the second sub-section we defined these processes and evaluate if the growth of the OSM street networks are also governed by these processes. In the final sub-section we examine the growth of the OSM street networks in terms of topology. We describe in detail the results corresponding to the Maynooth region and subsequently summaries those corresponding to the Waterford and Wicklow regions. 
a

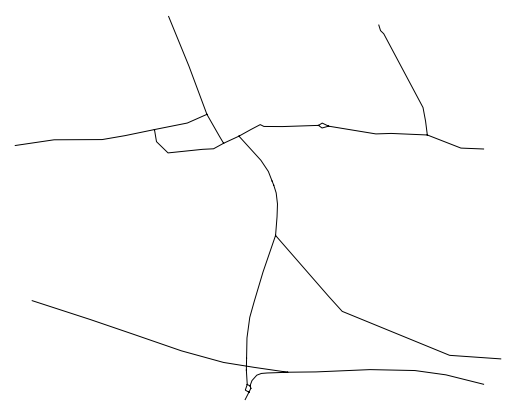

C

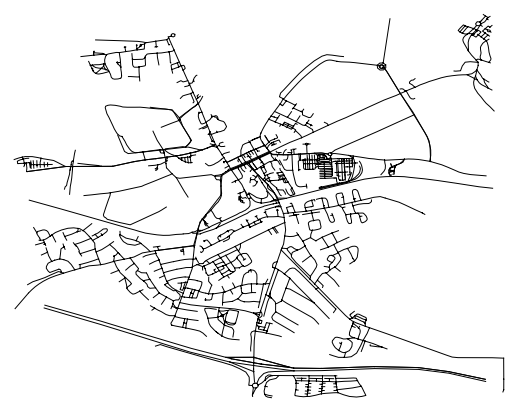

$\mathrm{e}$

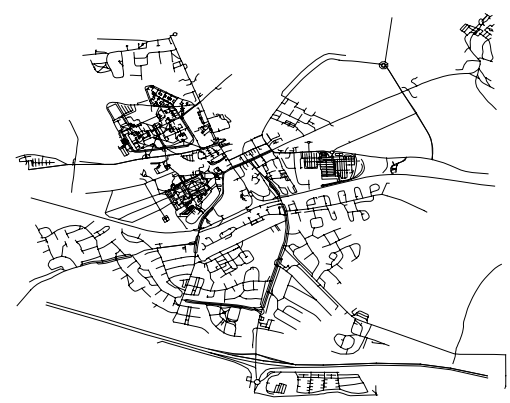

b

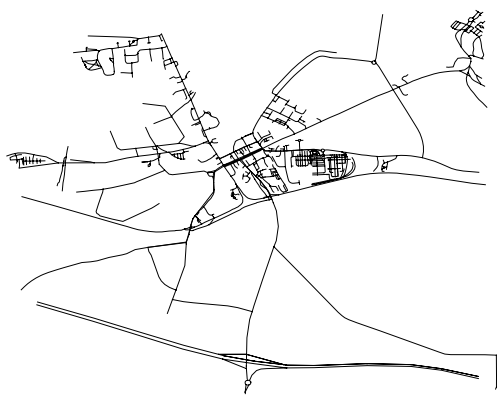

d

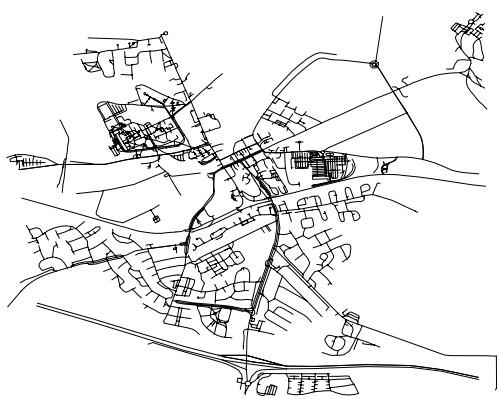

$\mathrm{f}$

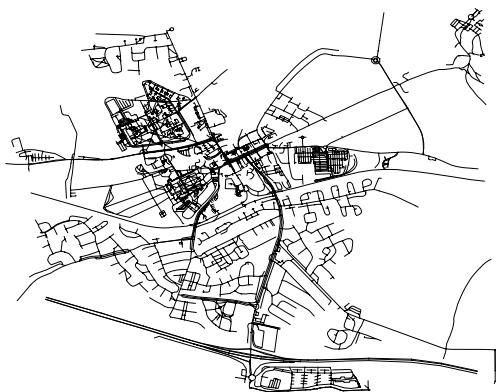

Fig. 1. OSM representations of the Maynooth street network on the dates 01-11-07, 01-02-09, 01-03-09, 01-07-09, 01-09-09 and 01-10-11 are displayed in (a), (b), (c), (d), (e) and (f) respectively.

\subsection{Rate of growth}

As illustrated by Fig. 1 the degree to which the Maynooth OSM street network grows during the study period is significant. Fig. 2 displays the value $\left|V_{i}\right|$ (number of graph vertices) for each version $i$ of the network. If we consider $\left|V_{i}\right|$ to be a measure of network size it is evident that the rate of network growth is not uniform and at a maximum during the middle of the study period. In order to remove rate of growth as a factor in our study we adopt the number of vertices $\left|V_{i}\right|$ as the internal clock of the process. Thus, we study the growth of the network as a function of $\left|V_{i}\right|$. This approach was also used by Strano et al. (2012) to remove a demographic factor when studying the growth of a street network.

Let $N_{i}$ be the set of OSM nodes representing version $i$ of the network. The value $\left|N_{i}\right|$ is therefore a measure of the corresponding OSM dataset size. Using this fact it is possible to show that the relationship between network growth and OSM dataset size is linear. This is demonstrated by 


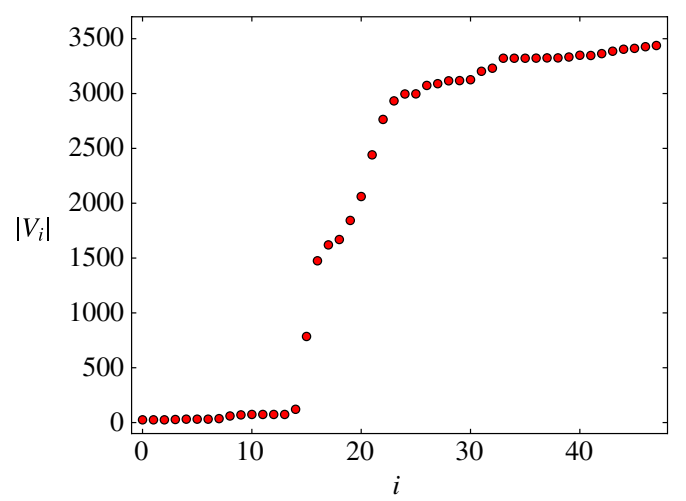

Fig. 2. Number of vertices for the Maynooth OSM street network.

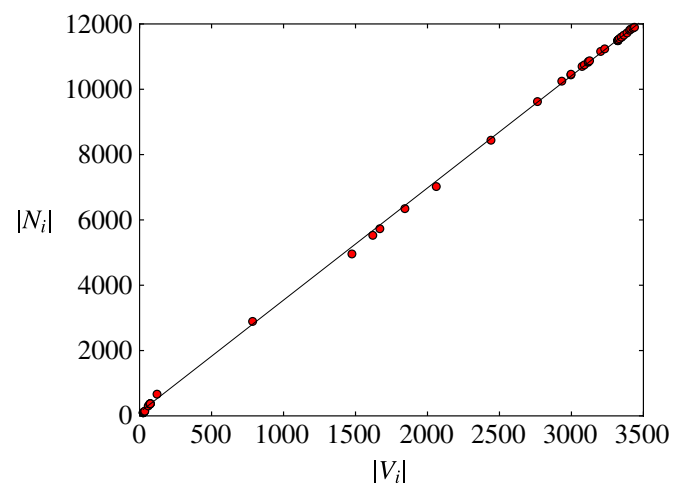

Fig. 3. Number of OSM nodes for the Maynooth OSM street network.

examining the plot of $\left|N_{i}\right|$ versus $\left|V_{i}\right|$ in Fig. 3. The first degree polynomial equation $\left|N_{i}\right|=3.45\left|V_{i}\right|+$ 57.22 is represented in this figure by a black solid line and models the relationship which exists between these variables.

\subsection{Densification and exploration}

Strano et al. (2012) examined the properties of new edges in the growth of the street network located in a region north of Milan Italy. The authors demonstrated that this growth was governed by two elementary spatial processes referred to as densification and exploration. In the remainder of this section we describe the methodology used by Strano et al. (2012) to illustrate these facts and subsequently we evaluate if the Maynooth OSM street network is also governed by these processes.

The growth of a street network can be analysed in terms of street centrality which measures the importance of individual streets in a given network. The betweenness centrality of an edge is adopted which is defined as the number of times the edge is contained in shortest paths and therefore is a measure of the edge's influence on shortest paths in the network. The betweenness centrality of an edge $e$ in the network $G=(V, E)$, denoted $b(e)$, is defined in Eq. (1) where $\sigma_{i j}$ is the number of shortest paths from vertex $i$ to $j$ and $\sigma_{i j}(e)$ is the number of shortest paths which contain $e$. The average betweenness centrality of the network is defined by Eq. (2).

$$
b(e)=\sum_{i \in V} \sum_{\substack{i \in V \\ j \neq i}} \frac{\sigma_{i j}(e)}{\sigma_{i j}}
$$



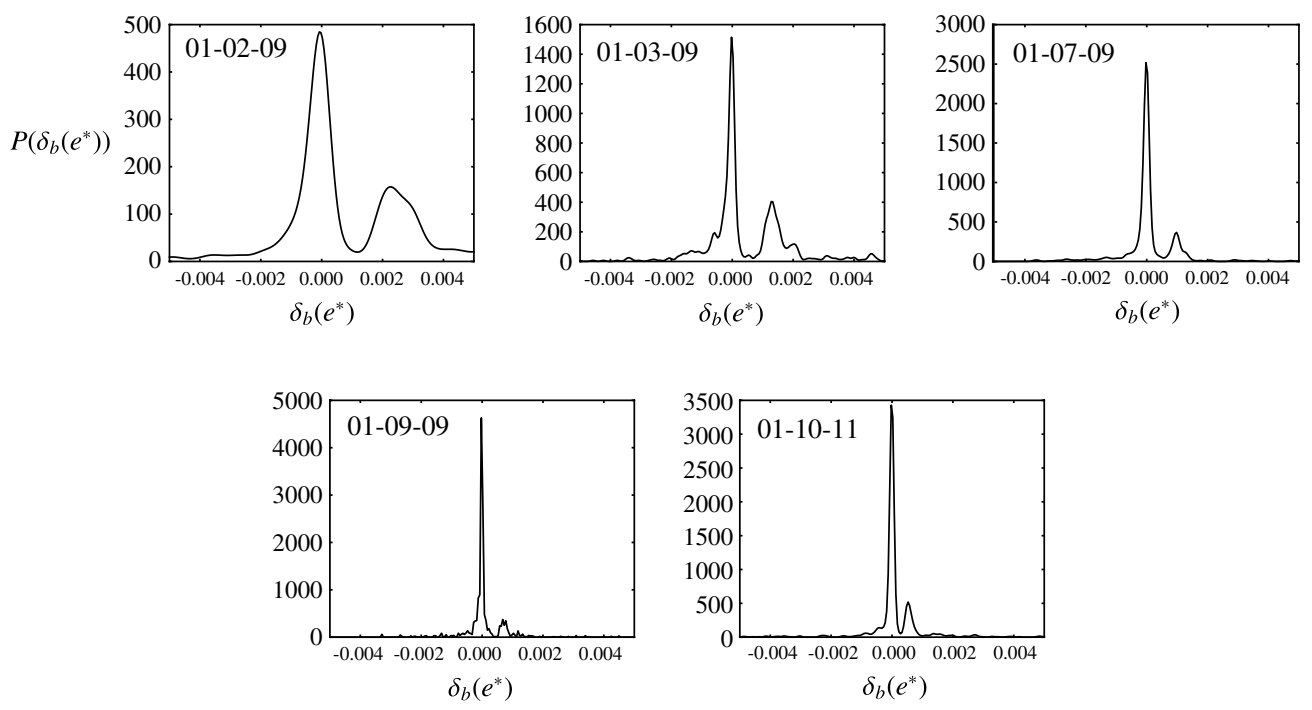

Fig. 4. Kernel density estimation of $\delta_{b}\left(e^{*}\right)$ for the Maynooth OSM street network.

$$
\bar{b}\left(G_{i}\right)=\frac{1}{\left(\left|V_{i}\right|-1\right)\left(\left|V_{i}\right|-2\right)} \sum_{e \in E_{i}} b(e)
$$

Let $\Delta E_{i}$ be the set of edges added to network $G_{i-1}$ to obtain $G_{i}$ and for $e^{*} \in \Delta E_{i}$ we denote $G_{i} \backslash\left\{e^{*}\right\}$ to be graph obtained by removing $e^{*}$ from $G_{i}$. The impact of an edge $e^{*}$ on the betweenness centrality of the network $G_{i}$, denoted $\delta_{b}\left(e^{*}\right)$, is defined in Eq. (3). The quantity $\delta_{b}\left(e^{*}\right)$ measures the relative variation of the network average betweenness centrality due to the removal of the edge $e^{*}$; that is, the degree to which its removal impacts on the average betweenness centrality. The computational complexity of computing $\delta_{b}\left(e^{*}\right)$ is $O\left(\left|V_{i}\right|\left|E_{i}\right|+\left|V_{i}\right|\left(\left|V_{i}\right|+\left|E_{i}\right|\right) \log \left|V_{i}\right|\right)$ (Brandes, 2001).

$$
\delta_{b}\left(e^{*}\right)=\frac{\bar{b}\left(G_{i}\right)-\bar{b}\left(G_{i} \backslash\left\{e^{*}\right\}\right)}{\bar{b}\left(G_{i}\right)}
$$

In order to study the properties of $\delta_{b}\left(e^{*}\right)$ in the context of the Maynooth region the six network versions corresponding to the dates 01-11-07, 01-02-09, 01-03-09, 01-07-09, 01-09-09 and 01-10-11 were selected. The number of vertices contained in these networks is 25, 785, 1475, 2061, 2764 and 3438 respectively. These specific network versions were chosen so that the values $\left|\Delta E_{i}\right|$ were similar and significantly large. All network edges on the date 01-11-07 are considered new and therefore this network version cannot be subject to analysis. A kernel density estimate of $\delta_{b}\left(e^{*}\right)$ values for each network version is displayed in Fig. 4. The distributions of $\delta_{b}\left(e^{*}\right)$ all exhibits two well separated peaks. The size of the left peak increases over time while the size of the right peak decreases over time and begins to merge with the left peak. To understand the nature and growth of these peaks we relate them to the corresponding street network. Fig. 5(a)-(e) displays the street network versions where edges corresponding to the left peaks of the kernel density estimates are coloured green and edges corresponding to the right peaks of the kernel density estimates are coloured red. A study of this figure reveals that new edges are divided into two classes. The green edges (left peak) tend to bridge existing streets creating new routes while the red edges (right peak) are usually cul-de-sacs. To quantify this characteristic of the two classes of edges we calculate the mean $k_{\min }\left(e^{*}\right)$ value for each class where $k_{\min }\left(e^{*}\right)$ is the minimum degree of the two end vertices of $e^{*}$. The mean $k_{\min }\left(e^{*}\right)$ values of the green edges in Fig. 1(b)-(e) are 3.1, 3.0, 3.2, 3.2 and 3.3 respectively. While the mean $k_{\min }\left(e^{*}\right)$ values of the red edges in Fig. 1 (b)-(e) are 1.2, 1.1, 1.2, 1.1 and 1.2 respectively.

These results correlate closely with those observed by Strano et al. (2012) in their analysis of a street network located in a region north of Milan. The authors suggest that this is a consequence of 
a

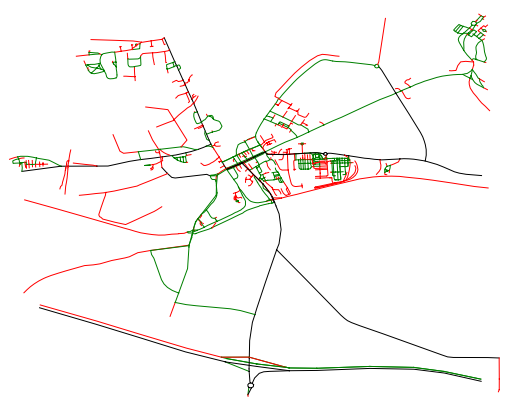

$\mathrm{c}$

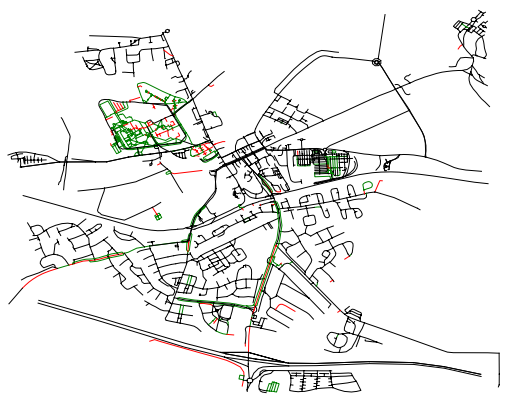

$\mathrm{b}$

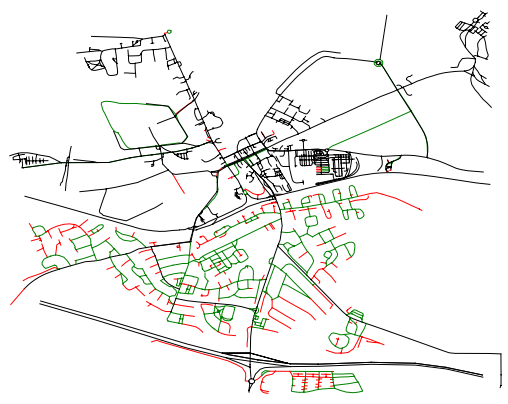

d

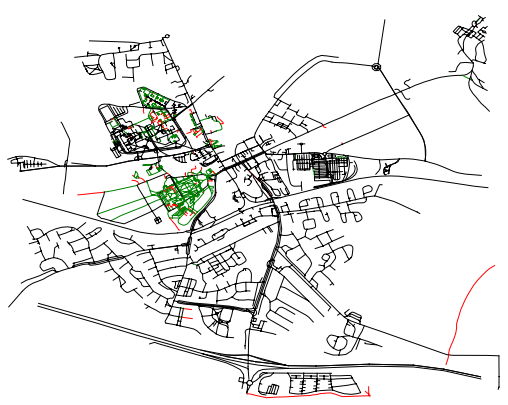

$\mathrm{e}$

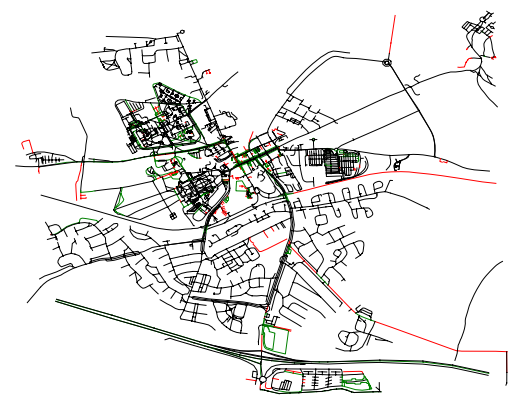

Fig. 5. The processes of densification (green) and exploration (red) exhibited by the Maynooth OSM street network growth on the dates 01-02-09, 01-03-09, 01-07-09, 01-09-09 and 01-10-11 are displayed in (a), (b), (c), (d) and (e) respectively.

the growth being subject to two distinct concurrent spatial processes. The first process of densification (green edges, left peak, $k_{\min }\left(e^{*}\right)>2$ ) is responsible for increasing the local density of the street network while the second process of exploration (red edges, right peak, $k_{\min }\left(e^{*}\right) \approx 1$ ) is responsible for the expansion of the network into new areas. This implies that the growth of the OSM street network in question is also be governed by both of these spatial processes. Strano et al. (2012) also suggested that in some cases both processes have a temporal sequence where exploration acts as a stimulation for densification. An examination of the top left regions of Fig. 5(a) and (c) reveals that this sequence can also occur in the context of OSM.

Although not presented in this paper, the two regions of Waterford and Wicklow also appear to be governed in a similar manner by densification and exploration. For example Fig. 6(a) displays the kernel density estimation of $\delta_{b}\left(e^{*}\right)$ for the Waterford region on $01-10-2009$. The processes of densification and exploration corresponding to this kernel density estimate are exhibited in Fig. 6(b). 
a

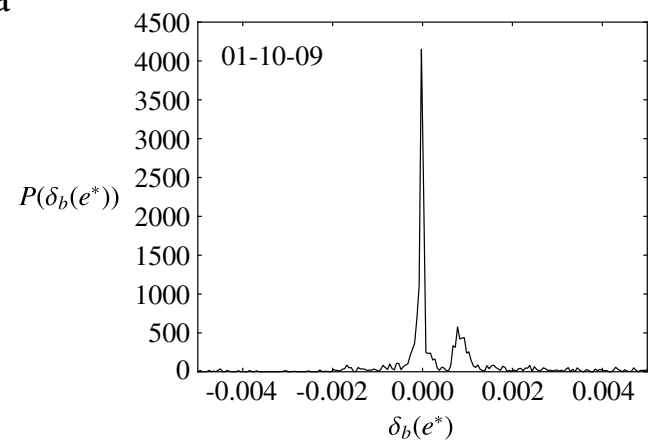

b

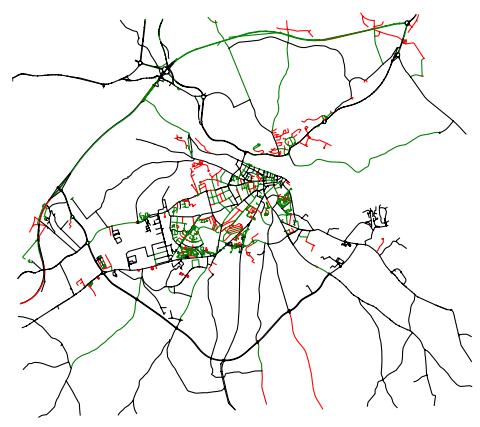

Fig. 6. Kernel density estimation of $\delta_{b}\left(e^{*}\right)$ for the Waterford OSM street network on 01-10-2009 is displayed in (a). The corresponding processes of densification (green) and exploration (red) are exhibited in (b).

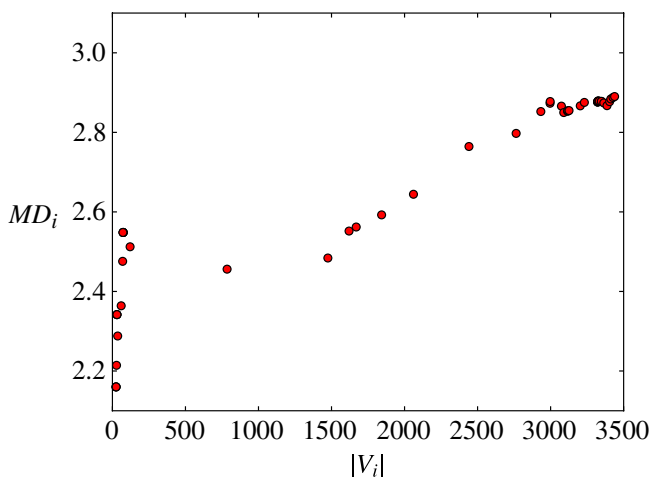

Fig. 7. Mean vertex degree for the Maynooth OSM street network.

\subsection{Network topology}

In this sub-section we examine the growth of the Maynooth OSM street network in terms of its topology. This is achieved by calculating summary statistics which describe the topology of the network in question. The degree distribution is a statistic commonly used to describe the topology of a given network (Chan et al., 2011). However the direct comparison of a large number of degree distributions is challenging and therefore a summary statistic of the degree distribution is used to allow a meaningful comparison (Strano et al., 2012). Also Barthelemy (2011) states that in the case of streets networks, and more generally spatial networks, the degree distribution is not a suitable approach for describing network topology and is of "little interest". As a summary statistic we use the mean vertex degree for each network version $i$ which we referred to as $M D_{i}$. This is computed using Eq. (4). Fig. 7 displays a plot of $M D_{i}$ versus $\left|V_{i}\right|$ which illustrates that the mean degree increases from 2.16 to 2.89 during the network growth. However the relationship between these variables is complex and non-linear.

$$
M D_{i}=\frac{2\left|E_{i}\right|}{\left|V_{i}\right|}
$$

Additional information regarding the network topology can be obtained using the alpha index ( $\alpha$ ) defined in Eq. (5). The alpha index measures the number of cycles in the network and in turn is a measure of network connectivity (Kansky, 1963; Levinson, 2012). It takes values in the interval [0, 1] where a value of 0 corresponds to a network with no circuits while a value of 1 corresponds to a 


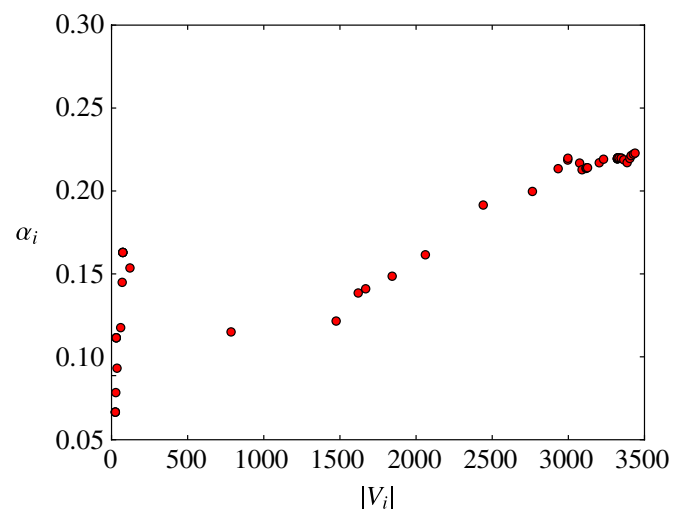

Fig. 8. Alpha index for the Maynooth OSM street network.

completely interconnected network. Fig. 8 displays a plot of $\alpha_{i}$ versus $\left|V_{i}\right|$ and illustrates that the number of cycles increases during the network growth. It is evident that the relationship existing between $\alpha_{i}$ and $\left|V_{i}\right|$ is correlated with the relationship existing between $M D_{i}$ and $\left|V_{i}\right|$. This is not surprising considering it has been previously demonstrated that many summary statistics of real world networks tend to be correlated (Jamakovic and Uhlig, 2008). The pattern that the mean vertex degree and alpha index both increase as the networks grows was also reflected in the parallel studies of the Waterford and Wexford regions.

$$
\alpha_{i}=\frac{\left|E_{i}\right|-\left|V_{i}\right|+1}{2\left|V_{i}\right|-5}
$$

The fact that densification dominates exploration to a greater degree as the network in each of the three regions grow explains the growth of the mean vertex degree and alpha index. That is, why both these topology summary statistics increase as the corresponding networks grow. Bridging existing streets through densification increases the number of cycles and the corresponding vertices introduced have degree greater than or equal to three. This represents the discovery of a novel link between the densification and exploration processes and network topology.

\section{Conclusions}

This article presents an analysis of the growth of three OSM street network representations located in Ireland and makes two main research contributions. Firstly we demonstrated that in each case the growth appears to be governed by the spatial processes of densification and exploration. Secondly we demonstrated that summary statistics of the network topology may be explained by the processes of densification and exploration. This represents the discovery of a novel link between different aspects of the growth.

The growth of an OSM street network is a function of contributor activity. As discussed in Section 2.1 the degree and nature of such activity exhibits significant spatial heterogeneity. Despite this fact, in this article, it has been demonstrated that the growth of OSM street networks corresponding to three independent regions appear to be governed by the same processes of densification and exploration. And that, in each case, as the network grows densification becomes more dominant relative to exploration. This suggests that the presence and nature of these processes may not be a function of contributor activity. Instead we believe that the presence and nature of these processes may be a consequence of the manner in which different networks in general grow. This is akin to the fact that many different networks exhibit small-world and/or scale-free properties (see Section 2.2). This theory is supported by the fact that Strano et al. (2012) previously showed these processes to govern the growth of a type of spatial network other than an OSM street network representation; that is, a street network in a region North of Milan. 
In the context of spatial networks one possible justification for this theory is the following. Only a finite amount of edges can be contained in a given spatial region and therefore, in order for a network to continue to grow, it must eventually expand into new regions through exploration. Once a region has been explored the network can continue to grow in that region through densification. This theory could also explain why, in all cases examined, as the spatial network grows densification becomes more dominant relative to exploration. Only a limited number of exploration streets may be added to a spatial region before it becomes completely explored. On the other hand, densification streets could be added to such a region long after this occurs.

It is possible that the processes of densification and exploration may also govern the growth of nonspatial networks. For example consider a social network where vertices represent people and an edge exists between two vertices if the people in question are friends. If people represented in the network make new friends with others not previously represented in the network this would correspond to exploration. On the other hand, if people represented in the network make new friends with others already represented in the network this would correspond to densification.

Not withstanding the good results obtained in our study, providing evidence that networks in general, and not just the given case studies, are governed by the processes of densification and exploration would require a larger scale study containing a greater number of networks of greater size. However the computational complexity of the corresponding algorithms presents an obstacle to this. As stated in Section 4.2 the computational complexity of computing $\delta_{b}\left(e^{*}\right)$ is significant and must be computed for every edge $e^{*}$. For example, computing the necessary results for the Maynooth region using a desktop machine with an Intel $2.8 \mathrm{GHz}$ dual core processor required roughly $5 \mathrm{~h}$. Therefore analysing larger scale networks presents a significant challenge in terms of time and resources. This could potentially be overcome using a distributed or GPU computing paradigm.

As discussed in Section 2.2 many different models of transportation network growth have been proposed. In this article we have examined the single model of Strano et al. (2012) and demonstrated that it can explain some aspects of the growth of OSM street networks. As part of future work we are interested in performing a parallel study using other models to determine if they provide any further insight into the OSM mapping process. For example, the model of Xie and Levinson (2009) considers the context of a transportation network growth which allows for the interaction between demand (travelers) and supply (transportation network). This model could also potentially be applied to OSM if one lets demand correspond to the number of active contributors in an area and supply correspond to the OSM representation in that area. As discussed in Section 2.1, it has previously been demonstrated that a relationship exists been the degree of contributor activity in an area and the corresponding OSM representation. In the introduction to this article we stated that understanding the processes governing the growth of OSM has the potential to serve as a platform for data quality assessment. However the question of how to link such a process to a methodology for quality assessment is still in many regards open and therefore represents another possible future research direction.

\section{Acknowledgements}

The research presented in this paper was primarily funded by the Irish Research Council (IRC) EMPOWER program. It was also in part funded by the Irish Environmental Protection Agency (EPA) STRIVE programme (Grant 2008-FS-DM-14-S4) and a Strategic Research Cluster Grant (07/SRC/I1168) from Science Foundation Ireland under the National Development Plan.

\section{References}

Barthelemy, M., 2011. Spatial networks. Physics Reports 499 (1-3), 1-101.

Barthelemy, M., Flammini, A., Modeling urban street patterns. Physical Review Letters.

Brandes, U., 2001. A faster algorithm for betweenness centrality. The Journal of Mathematical Sociology 25 (2), 163-177.

Buhl, J., Gautrais, J., Reeves, N., Sol, R.V., Valverde, S., Kuntz, P., Theraulaz, G., 2006. Topological patterns in street networks of self-organized urban settlements. The European Physical Journal B. Condensed Matter and Complex Systems 49, 513-522.

Chan, S., Donner, R., Lmmer, S., 2011. Urban road networks - spatial networks with universal geometric features? The European Physical Journal B. Condensed Matter and Complex Systems 84, 563-577.

Corcoran, P., Mooney, P., 2013. Characterising the metric and topological evolution of openstreetmap street network representations. The European Physical Journal Special Topics. 
Costa, L.d.F., Rodrigues, F.A., Travieso, G., Villas Boas, P.R., 2007. Characterization of complex networks: a survey of measurements. Advances in Physics 56 (1), 167-242.

Crucitti, P., Latora, V., Porta, S., 2006. Centrality measures in spatial networks of urban streets. Physical Review E 73, 036125.

Derrible, S., Kennedy, C., 2010. The complexity and robustness of metro networks. Physica A: Statistical Mechanics and its Applications 389 (17), 3678-3691.

Erath, A., Lochl, M., Axhausen, K., 2009. Graph-theoretical analysis of the swiss road and railway networks over time. Networks and Spatial Economics 9 (3), 379-400.

Ferber, C., Holovatch, T., Holovatch, Y., Palchykov, V., 2009. Modeling metropolis public transport. In: Appert-Rolland, C., Chevoir, F., Gondret, P., Lassarre, S. (Eds.), Traffic and Granulat Flow. Springer, pp. 709-720.

Girres, J., Touya, G., 2010. Quality assessment of the French openstreetmap dataset. Transactions in GIS 14 (4), 435-459.

Goodchild, M., 2007. Citizens as sensors: the world of volunteered geography. GeoJournal 69 (4), 211-221.

Goodchild, M., Glennon, J., 2010. Crowdsourcing geographic information for disaster response: a research frontier. International Journal of Digital Earth 3 (3), 231-241.

Goodchild, M., Li, L., 2012. Assuring the quality of volunteered geographic information. Spatial Statistics 1 (0), 110-120.

Haklay, M., 2010. How good is volunteered geographical information? a comparative study of openstreetmap and ordnance survey datasets. Environment and Planning B: Planning and Design 37 (4), 682-703.

Haklay, M., Basiouka, S., Antoniou, V., Ather, A., 2010. How many volunteers does it take to map an area well? the validity of linus; law to volunteered geographic information. The Cartographic Journal 47 (4), 315-322.

Holme, P., Saramaki, J., 2012. Temporal networks. arXiv:1108.1780v2.

Jamakovic, A., Uhlig, S., 2008. On the relationships between topological measures in real-world networks. Networks and Heterogeneous Media 3 (2), 345-358.

Jiang, B., Claramunt, C., 2004. Topological analysis of urban street networks. Environment and Planning B: Planning and Design $31(1), 151-162$.

Kansky, K.J., 1963. Structure of transportation networks: relationships between network geometry and regional characteristics, Thesis, University of Chicago.

Kuhn, W., 2007. Volunteered geographic information and GIScience. In: Workshop on Volunteered Geographic Information, No. 86-97, University of California, Santa Barbara.

Levinson, D., 2012. Network structure and city size. PLoS ONE 7 (1), e29721.

Mashhadi, A., Quattrone, G., Capra, L., 2013. Putting ubiquitous crowd-sourcing into context. In: ACM Conference on Computer Supported Cooperative Work and Social Computing, San Antonio, Texas.

Masucci, A.P., Smith, D., Crooks, A., Batty, M., 2009. Random planar graphs and the London street network. The European Physical Journal B. Condensed Matter and Complex Systems 71, 259-271.

Mooney, P., Corcoran, P., 2011. Accessing the history of objects in openstreetmap. In: AGILE International Conference on Geographic Information Science.

Mooney, P., Corcoran, P., Winstanley, A., 2010. Towards quality metrics for openstreetmap. In: Proceedings of the 18th SIGSPATIAL International Conference on Advances in Geographic Information Systems, New York, NY, USA, pp. 514-517.

Mulligann, C., Janowicz, K., Ye, M., Lee, W., 2011. Analyzing the spatial-semantic interaction of points of interest in volunteered geographic information. In: Proceedings of the 10th International Conference on Spatial Information Theory. COSIT'11. Springer-Verlag, Berlin, Heidelberg, pp. 350-370.

Neis, P., Zielstra, D., Zipf, A., 2012. The street network evolution of crowdsourced maps: openstreetmap in Germany $2007-2011$. Future Internet $4(1), 1-21$.

Neis, P., Zipf, A., 2012. Analyzing the contributor activity of a volunteered geographic information project - the case of openstreetmap. ISPRS International Journal of Geo-Information 1 (2), 146-165.

Panciera, K., Priedhorsky, R., Erickson, T., Terveen, L., 2010. Lurking? cyclopaths?: a quantitative lifecycle analysis of user behavior in a geowiki. In: Proceedings of the 28th International Conference on Human Factors in Computing Systems, New York, NY, USA, pp. 1917-1926.

Porta, S., Crucitti, P., Latora, V., 2006. The network analysis of urban streets: a dual approach. Physica A 853-866.

Roick, O., Hangenauer, J., Zipf, A., 2013. Osmatrix: Visualisation of contributions to osm, WWW document. http://koenigstuhl. geog.uni-heidelberg.de/osmatrix.

Strano, E., Nicosia, V., Latora, V., Porta, S., Barthelemy, M., 2012. Elementary processes governing the evolution of road networks. Scientific Reports 2, 296.

Trame, J., Keßler, C., 2011. Exploring the lineage of volunteered geographic information with heat maps. In: GeoViz.

Watts, D.J., Strogatz, S.H., 1998. Collective dynamics of 'small-world' networks. Nature 393 (6684), 440-442.

Xie, F., Levinson, D., 2009. Topological evolution of surface transportation networks. Computers, Environment and Urban Systems 33 (3), 211-223. 